TMUP-HEL-0017

hep-ph/0009019

\title{
A Two-Dimensional Model with Chiral Condensates and Cooper Pairs Having QCD-like Phase Structure
}

\author{
Alan Chodos \\ Department of Physics, Yale University, New Haven, CT 06520-8120, USA and American \\ Physical Society, One Physics Ellipse, College Park, MD 20740-3844 USA \\ Hisakazu Minakatal" \\ Department of Physics, Tokyo Metropolitan University \\ 1-1 Minami-Osawa, Hachioji, Tokyo 192-0397, Japan, and \\ Research Center for Cosmic Neutrinos, Institute for Cosmic Ray Research, \\ University of Tokyo, Kashiwa, Chiba 277-8582, Japan \\ Fred Cooper and Anupam Singh \\ Los Alamos National Laboratory, Los Alamos, NM 87545, USA \\ Wenjin Maof \\ Department of Physics, Boston College, Chestnut Hill, MA 02467, USA (September 2000)
}

\begin{abstract}
We describe how a generalization of the original Gross-Neveu model from $U(N)$ to $O(N)$ flavor symmetry leads to the appearance of a pairing condensate at high density, in agreement with the conjectured phenomenon of color
\end{abstract}

\footnotetext{
*E-mail: chodos@aps.org

†E-mail: minakata@phys.metro-u.ac.jp

${ }^{\ddagger}$ E-mail: cooper@schwinger.lanl.gov, singh@lanl.gov

§E-mail: maow@physics.bc.edu
} 
superconductivity in $(3+1)$-dimensional QCD. Moreover, the model displays a rich phase structure which closely resembles the one expected in two-flavor QCD. 


\section{INTRODUCTION}

The work that will be described in this note had its origin at the beginning of the history of TMU-Yale collaboration about a decade ago, when one of us (A.C.) was working on QED with a chemical potential [1,2] and another (H.M.) suggested that the study of the Gross-Neveu model [3] with a chemical potential might have interesting applications to doped polyacetylene. This resulted in a pair of papers, in the first of which [4] the effective potential for the Gross-Neveu model was computed with a non-vanishing chemical potential $\mu$ at leading order in large $N$. In agreement with earlier work [5], a first-order phase transition was found at $\mu=m / \sqrt{2}$, where $m$ is the fermion mass, and this result was successfully compared to the phenomenology of doped polyacetylene [4,6]. In the second paper [7], the thermodynamic Bethe ansatz was employed to compute the next-to-leading $1 / N$ correction to the position of the phase-transition.

But the Gross-Neveu model, possessing aymptotic freedom and spontaneous chiral symmetry breaking, was originally introduced as an analogue of QCD. With renewed interest in QCD at finite density [8], the TMU-Yale collaboration shifted its focus to trying to understand the phenomenon of color superconductivity. Before describing that work, we make a few remarks about the role played by the large $N$ approximation, which will be used throughout this paper, in the prediction of the superconducting phase transition.

The issue is the following: the superconducting phase will involve a non-vanishing pairing condensate, which spontaneously breaks the $U(1)$ of fermion number. But the generalized Gross-Neveu model is a $1+1$ dimensional theory, and theorems exist [9] that forbid the spontaneous breaking of continuous symmetries in $1+1$ dimensions. Nevertheless, to leading order in $1 / N$ one can compute the effective potential, and one finds that the condensate indeed forms. As Witten has explained [10], corrections are expected beyond the leading order which will remove the condensate. More explicitly, the vacuum expectation value of $B(x)$ must vanish at any finite $N$ because its correlation function $\left\langle B(x) B^{\dagger}(y)\right\rangle$ will fall off, for large $(x-y)$, as $|x-y|^{-c / N}$. If we take the limit $N \rightarrow \infty$ first before taking the limit $|x-y| \rightarrow \infty$ it implies non-vanishing vacuum expectation value of $B(x)$. 
One can enhance one's intuition on this matter if one accepts the analogy between the infinite number of fields that are present in the large $N$ approximation and the infinite set of fields one obtains upon making a Kaluza-Klein reduction from a higher number of dimensions. Admittedly, this analogy is not perfect, because in the large $N$ approximation the fields are all identical, whereas in Kaluza-Klein they typically represent a tower of states with indefinitely increasing masses. Nevertheless, the analogy suggests that the leading $N$ theory somehow shares the physics of a theory in a higher number of dimensions, where the no-go theorems do not apply. The analogy further suggests that if one is interested primarily in elucidating properties of $(3+1)$-dimensional QCD, the large $N$ approximation to the Gross-Neveu model may be a more appropriate object of study than the full theory.

Even more relevant examples in this context are provided by the Eguchi-Kawai model [11], or by a class of large-N matrix models [12,13]. In these matrix models the eigenvalues of the matrix are somehow converted, in the large- $\mathrm{N}$ limit, into the spatial dimensions. Thus, the matrix models in zero dimension thereby serve as definitions of 4-dimensional YangMills and higher-dimensional superstring or supergravity theories. It is not clear to us if the analogy goes through to the vector-like large $N$ theories such as ours. Nonetheless, it is at least suggestive enough to lead to the speculation that Coleman's theorem is violated in $(1+1)$-dimensional large $N$ theories because they are actually the theories in higher dimensions.

\section{THE $O(N)$ GROSS-NEVEU MODEL}

The phase structure of the original Gross-Neveu model is well-known [5]: at low temperature and density, the chiral symmetry is broken; as either is increased, the chiral condensate vanishes and the symmetry is restored. But there is no evidence for a second, pairing condensate that would be the analogue of color superconductivity in QCD. It is necessary to add to the original model an extra term in the Lagrangian which, as it turns out, has a simple interpretation. The original model, with $N$ identical fermi fields, possesses $U(N)$ symmetry. The extra term is what can be added if one demands only $O(N)$ symmetry in 
flavor space.

Explicitly, our lagrange density reads

$$
\begin{aligned}
\mathcal{L} & =\bar{\psi}^{(i)} i \not \bar{\psi} \psi^{(i)}+\frac{1}{2} g^{2}\left[\bar{\psi}^{(i)} \psi^{(i)}\right]\left[\bar{\psi}^{(j)} \psi^{(j)}\right] \\
& +2 G^{2}\left(\bar{\psi}^{(i)} \gamma_{5} \psi^{(j)}\right)\left(\bar{\psi}^{(i)} \gamma_{5} \psi^{(j)}\right)-\mu \psi^{\dagger(i)} \psi^{(i)} .
\end{aligned}
$$

The flavor indices $i, j$ are summed from 1 to $N$. The first 2 terms are the original $G N$ model. The third term, because of the way the flavor indices are summed, is $O(N)$ but not $U(N)$ invariant. The last term represents the chemical potential. The gamma matrices are $2 \times 2$, and we choose $\gamma^{0}=\sigma_{1}, \gamma^{1}=-i \sigma_{2} ; \gamma_{5}=\sigma_{3}$. Then

$$
2 G^{2} \bar{\psi}^{(i)} \gamma_{5} \psi^{(j)} \bar{\psi}^{(i)} \gamma_{5} \psi^{(j)}=-G^{2}\left[\epsilon_{\alpha \beta} \psi_{\alpha}^{\dagger(i)} \psi_{\beta}^{\dagger(i)}\right]\left[\epsilon_{\gamma \delta} \psi_{\gamma}^{(j)} \psi_{\delta}^{(j)}\right]
$$

Following standard techniques [14] we introduce auxiliary fields:

$$
\begin{aligned}
& m(x)=-g^{2} \bar{\psi} \psi \\
& B(x)=-G^{2} \epsilon_{\alpha \beta} \psi_{\alpha}^{(i)} \psi_{\beta}^{(i)}
\end{aligned}
$$

and integrate out the fermions to obtain the effective action $\Gamma_{\text {eff }}$ as a function of $m$ and $B$. This is described in more detail in refs. [15, [16].

As appropriate to a vacuum solution, we assume that $m$ and $B$ are constants, and write

$$
\Gamma_{e f f}=-N \int\left(d^{2} x\right) V_{e f f}
$$

We set $g^{2} N=\lambda$, and $G^{2} N=\kappa / 4$. The large $N$ limit is defined by letting $N \rightarrow \infty$ with $\lambda$ and $\kappa$ fixed. We find

$$
V_{e f f}(m, M)=\frac{m^{2}}{2 \lambda}+\frac{M^{2}}{2 \kappa}+V_{e f f}^{(1)}(m, M)
$$

where $M^{2}=4 B^{\dagger} B$. Explicit computation yields 16

$$
V_{e f f}^{(1)}(m, M)=-\frac{1}{2 \pi} \int_{0}^{\Lambda} d k\left[k_{+}+k_{-}+\frac{2}{\beta} \ln \left(1+e^{-\beta k_{+}}\right)+\frac{2}{\beta} \ln \left(1+e^{-\beta k_{-}}\right)\right]
$$


where $\Lambda$ is an ultraviolet cutoff, $\beta=1 / k T$ ( $\mathrm{T}=$ temperature) and

$$
k_{ \pm}=\sqrt{b_{1} \pm 2 b_{2}}
$$

with

$$
b_{1}=M^{2}+m^{2}+\mu^{2}+k^{2}
$$

and

$$
b_{2}=\left[M^{2} m^{2}+\mu^{2}\left(k^{2}+m^{2}\right)\right]^{1 / 2} .
$$

\section{RENORMALIZATION}

As with the original GN model, we renormalize the theory, thereby absorbing the dependence on $\Lambda$ in the relation between the bare couplings $\lambda$ and $\kappa$ and their renormalized counterparts $\lambda_{R}$ and $\kappa_{R}$. Recall that in the original model, one trades the dependence on $\Lambda$ for dependence on a renormalization scale $m_{0}$, or equivalently on the fermion mass $m_{F}$. Once one fixes $m_{F}$ at its physical value, there are no further parameters in the theory: the renormalized coupling $\lambda_{R}$ varies with $m_{0}$ in such a way as to keep $m_{F}$ fixed.

Similar features appear in the more general model, except that now there are two renormalized couplings. As a consequence, the theory has two physical parameters: the fermion mass $m_{F}$, and another parameter $\delta$ that is independent of the choice of renormalization scale, and is defined by

$$
\delta=\frac{1}{\kappa_{R}}-\frac{1}{2 \lambda_{R}}=\frac{1}{\kappa}-\frac{1}{2 \lambda}
$$

(Although $\kappa$ and $\lambda$ separately depend on the cutoff $\Lambda$, the combination represented by $\delta$ does not.) Further computation [16] allows us to express the renormalized $V_{\text {eff }}$ as a function of the condensates $m$ and $M$, the physical parameters $m_{F}$ and $\delta$, and the temperature $T$ and chemical potential $\mu$ : 


$$
\begin{aligned}
V_{e f f}=\delta M^{2} & -\frac{1}{2 \pi} \int_{0}^{\infty} d k\left[k_{+}+k_{-}+\frac{2}{\beta}\left(\ln \left(1+e^{-\beta k_{+}}\right)+\ln \left(1+e^{-\beta k_{-}}\right)\right)-2 k_{1}\right. \\
& \left.-2 k\left(m^{2}+M^{2}\right) \frac{1}{\sqrt{k^{2}+m_{F}^{2}}}\right] .
\end{aligned}
$$

This expression is valid for $\delta>0$. For $\delta<0$, there is no fermion mass, and instead at $\mu=T=0$ there is a pairing condensate $M^{2}=\Delta^{2}$. Since QCD has broken chiral symmetry but no pairing condensate at $\mu=T=0$, we shall restrict ourselves to $\delta>0$ henceforth. By studying how $\lambda_{R}$ and $\kappa_{R}$ vary with renormalization scale, one sees that both are asymptotically free. (Because $\delta$ is independent of this scale, it follows that if one of these couplings is asymptotically free so must the other be.)

\section{PHASE STRUCTURE}

The phase structure of the theory can now be mapped out by fixing $m_{F}, \delta, \mu$ and $T$, and finding the minimum of $V_{\text {eff }}$ with respect to $m$ and $M$. If $m=M=0$, the theory is fully symmetric; if $m \neq 0$, chiral symmetry is spontaneously broken, while if $M \neq 0$, there is a pairing condensate which represents the analogue of color superconductivity.

Because of the complexity of $V_{\text {eff }}$, this analysis must be done numerically. The results are schematically displayed in Figure 1 for a typical value of $\delta$. 


\section{FIGURES}

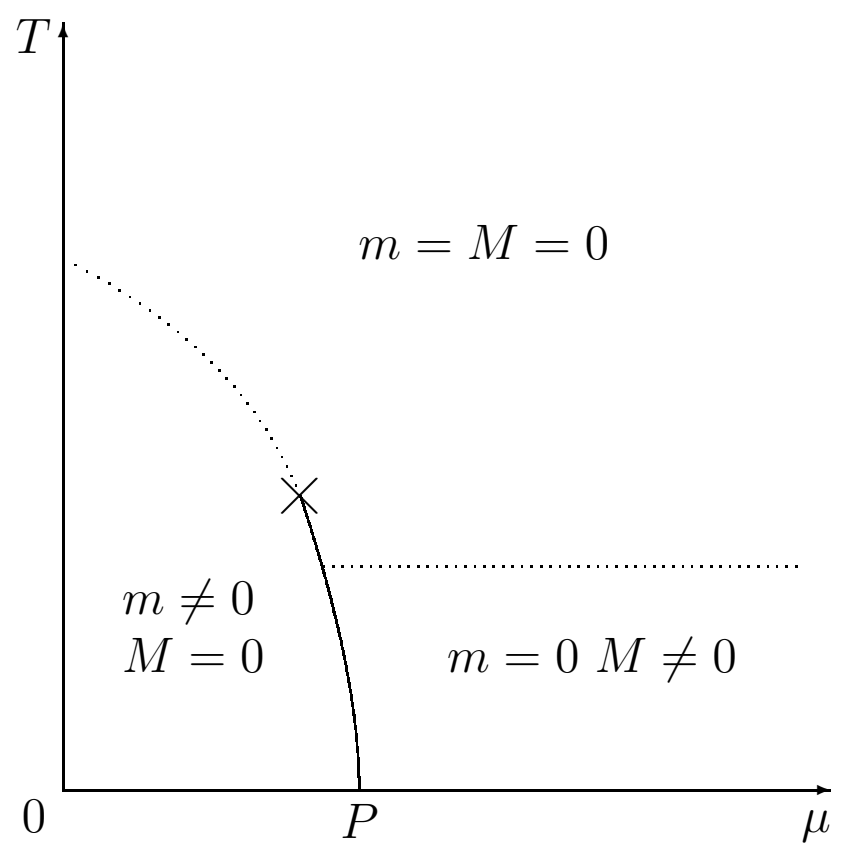

FIG. 1. A schematic illustration of the phase diagram on $T-\mu$ plane.

The point $\times$ is a tricritical point, at which the order of the phase transition changes from first (solid line) to second (dotted line). The point $P$ is located at $\mu^{2}=\frac{m_{F}^{2}}{2}\left[1-e^{-4 \pi \delta}\right]$. This generalizes the earlier result for the $U(N)$ GN model, $\mu^{2}=\frac{1}{2} m_{F}^{2}$, for which $\delta \rightarrow \infty$. Note that, interestingly, even though we have asymptotic freedom, the $M \neq 0$ phase persists for arbitrarily large $\mu$. In fact, the transition temperature from the Cooper pairing phase to the unbroken phase is independent of $\mu$.

The figure is qualitatively valid for

$$
\delta>\delta_{c} \cong \frac{1.13}{4 \pi}
$$

For smaller, positive $\delta$, the tricritical point disappears and the phase transition for fixed $\mu$ is always second order. For $\delta<0$ the chirally broken phase disappears, and there is only a pairing phase at low $T$ and an unbroken phase at high $T$.

There is no case of a phase in which both $m$ and $M$ are non-zero. However, there can be coexistence of the $m \neq 0$ and $M \neq 0$ phases along the line of first-order phase transitions. 


\section{CONCLUSIONS}

The phase structure displayed in Figure 1 is strikingly similar to that conjectured in the literature for two-flavor QCD. This gives us confidence that further properties of QCD can perhaps be studied reliably in this $1+1$-dimensional theory, whereas calculations involving the full 3+1-dimensional QCD may be prohibitively difficult. For example, work is in progress [17] on dynamical calculations, in which the system is prepared initially in a dense, hot state and allowed to cool and expand. Various distributions, such as the "pion" wave function and the "pion" correlation function, are followed as this evolution takes place. So far these computations have been performed only in the $U(N)$ model, and in the approximation that the expectation value of physical quantities depends only on proper time $\tau=\sqrt{t^{2}-z^{2}}$, but more general results are expected in future investigations.

\section{ACKNOWLEDGEMENTS}

We wish to thank Gregg Gallatin for interesting conversations. The research of AC is supported in part by DOE grant DE-FG02-92ER-40704. The research of FC and AS is supported by the DOE. In addition, AC and HM are supported in part by the Grantin-Aid for International Scientific Research No. 09045036, Inter-University Cooperative Research, Ministry of Education, Science, Sports and Culture of Japan. This work has been performed as an activity supported by the TMU-Yale Agreement on Exchange of Scholars and Collaborations. FC and HM are grateful for the hospitality of the Center for Theoretical Physics at Yale. HM, AC, and WM are grateful for the hospitality of the theory group at Los Alamos. In addition, AC wishes to express his gratitude for the opportunity to present these results at the TMU-Yale Symposium. 


\section{REFERENCES}

[1] A. Chodos, K. Everding and D.A. Owen, Phys. Rev. D42 (1990) 2881.

[2] A. Chodos, in "Vacuum Structure in Intense Fields," H.M. Fried and B. Mueller, eds., Plenum Press, New York (1991), p. 177.

[3] D. J. Gross and A. Neveu, Phys. Rev. D10 (1974) 3235.

[4] A. Chodos and H. Minakata, Phys. Lett. A191 (1994) 39.

[5] U. Wolff, Phys. Lett. B 157 (1985) 303; L. Jacobs, Phys. Rev. D10 (1974) 3945; B. Harrington and A. Yildiz, Phys. Rev. D11 (1975) 779; K.G. Klimenko, Teor. Mat. Fiz. 75 (1988) 226.

[6] H. Minakata and A. Chodos, in Recent Developements in Nonperturbative Quantum Field Theory: Proceedings, Edited by Y. M. Cho and M. Virasoro, World Scientific, Singapore, 1998.

[7] A. Chodos and H. Minakata, Nucl. Phys. B490 (1997) 687.

[8] D. Bailin and A. Love, Phys. Rep. 107 (1984) 325; M. Iwasaki and T. Iwado, Phys. Lett. 350B (1995) 163; M. Alford, K. Rajagopal and F. Wilczek, Phys. Lett. 422B (1998) 247; R. Rapp, T. Schäfer, E.V. Shuryak and M. Velkovsky, Phys. Rev. Lett. 81 (1998) 53.

[9] S. Coleman, Commun. Math. Phys. 31 (1973) 259; N.D. Mermin and H. Wagner, Phys. Rev. Lett. 17 (1966) 1133.

[10] E. Witten, Nucl. Phys. B145 (1978) 110.

[11] T. Eguchi and H. Kawai, Phys. Rev. Lett. 48 (1982) 1063.

[12] N. Ishibashi, H. Kawai, Y. Kitazawa, and A. Tsuchiya, Nucl. Phys. B498 (1997) 467.

[13] T. Banks, W. Fischler, S. H. Shenker, and L. Susskind, Phys. Rev. D55 (1997) 5112.

[14] J. Hubbard, Phys. Rev. Lett. 3 (1959) 77; R.L. Stratonovich, Doklady Akad. Nauk. 
SSSR 115 (1957) 1097; S. Coleman, Aspects of Symmetry, Cambridge Press, 1985, p. 354.

[15] A. Chodos, H. Minakata and F. Cooper, Phys. Lett. B449 (1999) 260.

[16] A. Chodos, F. Cooper, W.-J. Mao, H. Minakata and A. Singh, Phys. Rev. D61 (2000) 045011.

[17] W.-J. Mao, F. Cooper, A. Singh, and A. Chodos, hep-ph/0007244. 